\title{
Frontal fibrosing alopecia and genital Lichen sclerosus: single-center experience
}

Sara Grassi, MD, ${ }^{1}$ Giulia Tadiotto Cicogna, MD ${ }^{2}$ Francesca Magri, MD, ${ }^{1}$ Maria Caterina Fortuna, MD,${ }^{1}$ Gemma Caro, MD,${ }^{1}$ Angelina Pernazza, MD,${ }^{3}$ Giuseppe Soda, MD,${ }^{3}$ Emanuele Miraglia, MD, ${ }^{1}$ Sandra Giustini, MD, PhD, ${ }^{1}$ Marta Carlesimo, MD,${ }^{1}$ Alfredo Rossi, MD, $\mathrm{PhD}{ }^{1}$

${ }^{1}$ Department of Internal Medicine and Medical Specialties, Dermatology, Sapienza University of Rome, Rome, Italy

${ }^{2}$ Unit of Dermatology, University of Padua, Padua, Italy

${ }^{3}$ Department of Molecular Medicine, Sapienza University of Rome, Rome, Italy

Running head: Genital Lichen sclerosus in FFA patients

Abstract count: 239

Text count: 2376

Figures: 2

Tables: 0

References: 25

Corresponding author:

Sara Grassi, M.D.

Department of Internal Medicine and Medical Specialties,

"Sapienza" University of Rome,

Piazzale Aldo Moro, 5, 00185 Roma RM

e-mail sara.grassi03@gmail.com

phone number: 00393293034802

Funding Sources: None

Conflict of Interest: none

Role of Sponsor: NA

Prior Presentation: There is no prior presentation of this work.

Acknowledgements: None

This article has been accepted for publication and undergone full peer review but has not been through the copyediting, typesetting, pagination and proofreading process, which may lead to differences between this version and the Version of Record. Please cite this article as doi: $\underline{10.1111 / J O C D .13573}$

This article is protected by copyright. All rights reserved 
Keywords: Frontal fibrosing alopecia; scarring alopecia; ECM 1; trichoscopy; comorbidities; Lichen sclerosus.

DR. SARA GRASSI (Orcid ID : 0000-0001-8547-8393)

DR. GEMMA CARO (Orcid ID : 0000-0002-6568-3389)

Article type : Original Contribution

\section{Abstract}

Background: Despite the incidence of Frontal fibrosing alopecia (FFA) has been increasing in last two decades, the pathophysiology and trigger factors of FFA have not been yet fully understood.

Aims: The aim of this study was to describe epidemiology, clinical and trichoscopic features and comorbidities of FFA patients, in order to improve the understanding of this disease.

Patients/Methods: A retrospective, observational monocentric study was conducted from 2003 to 2019. Data concerning epidemiology (age, gender, age of menopause and age of FFA onset), comorbidities, current therapies, localization of FFA (such as frontotemporal hairline, occipital, eyebrow, eyelash, beard, sideburns and body hair), presence of papules and sign of Lichen planus (LP) at skin, mucosae and/or nail were collected for each patient included.

Results: A total of 119 Caucasian, adult patients ( 8 men, 111 female) with FFA were enrolled in the study. Cutaneous, mucosal or nail localization of LP were found in $16 \%$ of our subjects. Interestingly, 15 out of 119 subjects $(10.61 \%)$ were affected by concomitant genital Lichen sclerosus (LS) and 5 out of these 15 patients (4.38\%) presented both LS and LP in association with FFA

Conclusion: Considering the high prevalence of LS in FFA patients in our case series, and the frequency of autoimmune comorbidities in both LS and FFA, it is possible to hypothesize an autoimmune process in both conditions. Further studies are needed for a better understanding of the nature of the association between LS and FFA. 


\section{INTRODUCTION}

Frontal fibrosing alopecia (FFA) is a scarring alopecia described for the first time in 1994 by Kossard S. ${ }^{1}$ FFA affects prevalently postmenopausal women, even though premenopausal women and men may also be involved. ${ }^{2}$

Despite the incidence of FFA has been increasing in recent years, the pathophysiology and triggers factors of FFA have not been yet fully understood. Genetic predisposition (such as HLA-B*07:02), ${ }^{3}$ as well as hormonal factors and immune dysfunction appears to be implicated in the onset of FFA. Recently, environmental factors, such as the use of sunscreens and other cosmetic compounds, have been also hypothesized to play a role in FFA but it was not conclusively proven yet. ${ }^{4}$

Natural history of FFA has also not been completely understood and treatment is not standardized although an improvement or a stabilization with corticosteroids, tacrolimus, 5alpha-reductase inhibitors and oral immunomodulators have been reported in the Literature. ${ }^{5}$ For these reasons and because of the increasing incidence of FFA in recent years, it is crucial to collect data and to report them in detail, as recently suggested by several Authors. ${ }^{5}$

The aim of this study was to describe epidemiology, clinical and trichoscopic features and comorbidities of FFA patients, in order to improve the understanding of this disease.

\section{METHODS}

A retrospective, observational monocentric study was conducted from 2003 to 2019 at the Dermatology Department of Policlinico Umberto I in Rome. All adult patients affected by FFA were included in the study.

For each patient enrolled, medical records, laboratory data and trichoscopic pictures (FotoFinder medicam 1000, Germany) were reviewed to collect data concerning: epidemiology (age, gender, age of menopause and age of FFA onset), comorbidities, current therapies, localization of FFA (such as frontotemporal hairline, occipital, eyebrow, eyelash, beard, sideburns and body hair), presence of papules and sign of Lichen planus (LP) at skin, mucosae and/or nail. All doubtful cases of FFA have been confirmed histologically.

Furthermore, also trichoscopic features, the association with other types of alopecia, blood work-up (including thyroid hormones, antithyroid antibodies and antinuclear antibodies) and the associated symptoms (pruritus, trichodynia) were analyzed.

Eventually, clinical severity was classified in 5 grades using the Frontal Fibrosing Alopecia Severity Index (FFASI). ${ }^{6}$

This article is protected by copyright. All rights reserved 


\section{RESULTS}

A total of 119 Caucasian, adult patients (8 men, 111 female) with FFA were enrolled in the study. The average age was of 67 years in women and of 59 years in male. In $9.0 \%$ (10 women), FFA onset during fertile age, whereas in the majority $(n=101,91.0 \%)$ of our patients, symptoms and clinical features began in postmenopausal age (mean age at menopause onset: of 50.9 years).

The majority of patients were diagnosed of FFA after $2010(n=84,70.6 \%)$, whereas our first case of man with FFA date back to 2014.

Four out of 119 patients (3.5\%) reported familiarity for FFA with a sibling affected.

\section{Clinical features}

According to FFASI, 46 patients (38.6\%) was grade I and II, while group III counted 20 patients (17.0\%), group IV encompassed 5 patients (4.2\%), while group V included only 2 patients $(1.7 \%)$.

In 30 patients unusual pattern of presentation of FFA were found; in particular, among female patients AGA-like pattern was observed in 9 patients, cockade-like pattern in 2 patients, and ophiasis- like pattern in 14. Moreover, 5 male patients show "zig zag" pattern; beard and sideburns were involved in $25 \%$ of male patients.

Hair loss eyebrow (madarosis) was found in the $61.3 \%(n=73)$ of patients, with partial $(n=15$, $12.6 \%$, Figure $1 \mathrm{a}-\mathrm{d})$ or total loss $(\mathrm{n}=58,48.7 \%$, Figure 1 e-f). Eyelash alopecia was present only in 3 postmenopausal women $(2.5 \%)$ not affected by other types of alopecia.

\section{Trichoscopic findings}

Trichoscopical examination revealed the same features in patients with typical pattern as unusual patterns; in particular, the most frequent trichoscopic features were absence of follicular ostia and empty follicles which were found in all patients (100\%). Common signs were also perifollicular erythema and hyperkeratosis, present respectively in $63.0 \%(n=75)$ and $55.5 \%(n=66)$, moreover in $54,6 \%(n=65)$ of patients lonely hair there observed.

Surprisingly, facial papules were present only in 3 patients (2.5\%), 1 man and 2 women. Symptoms included itch, which was reported in 48 patients (40.3\%), and trichodynia, referred by 3 patients (2.5\%). Pain was not complained by any subject.

\section{Histopathological features}


Histopathological examination was performed on a total of 64 samples obtained in the frontal hairline in presence of clinical and trichoscopical signs of activity of the disease. All the patients were not receiving any treatment for at least 4 weeks before the skin biopsy.

Mild lymphohistiocytic inflammatory infiltrate and lamellar fibrosis mainly located at the isthmus and infundibulum area of the hair follicle were observed in all the samples (Figure 2 A, B, D). Most of the follicles involved were vellus-like. Apoptosis was prominent at the isthmus. In 10 samples, a perivascular lymphohistocytic infiltrate was also observed in the dermis, in absence of signs of vasculitis. Mean CD4/CD8 ratio was 1.64.

\section{Comorbidities}

Thyropathy was the most frequent associated diseases present in our patients. In fact, 21 out of 119 patients $(18.6 \%)$ were affected by hypothyroidism. Arterial hypertension was present in 19 out of 119 subjects (16.0\%) while hypercholesterolemia in 17, (14.3\%).

Concomitant Androgenic alopecia (AGA) or Female pattern hair loss (FPHL) were present in 19 patients (16.0\%) with FFA; finally, 5 patients ( 1 male and 4 female) were affected by Alopecia areata (AA). Also skin, mucosal or nail localization of LP were found in $16 \%$ of our subjects. Interestingly, 15 out of 119 subjects (10.61\%) were affected by concomitant genital Lichen sclerosus (LS), in particular 2 male patients and 13 female patients (Figure 2 C). Moreover, 5 female patients out of these 15 patients (4.38\%) presented both LS and LP in association with FFA. In particular, 5 patients affected by both diseases had grade I FFA, 2 patients had grade II, 4 grade III, and 1 grade IV.

\section{DISCUSSION}

FFA is a scarring alopecia whose incidence seems to be increasing in the last decade; ${ }^{5}$ also in our case series (collected over a 16 years' period), the majority of subjects (70.6\%) were enrolled after 2010. About $93 \%$ of all the patients were postmenopausal female (mean age: 67 years old), as expected. Several Authors have assumed a relationship between hormonal postmenopausal imbalance and the development of FFA, but currently this hypothesis has not been validated. Average age at diagnosis in our sample was significantly lower in male patients (59 years old) than female (67 years old), and in some case series it was reported to be even lower. ${ }^{8}$

Familiarity for FFA was present in $3.5 \%$ of our patients, concordant with the Literature. ${ }^{9}$

\section{FFA: clinical presentation}

This article is protected by copyright. All rights reserved 
To classify severity of FFA, we used the FFASI and we divided them in 5 groups, which corresponded to the 5 grades of severity of the pathology. ${ }^{6}$

Partial and total eyebrows loss were present in $12.6 \%$ and $48.7 \%$ respectively but this percentage seems to increase with the severity degree. In fact, in the first group total loss was present in the $31.1 \%$, in the second in $55.5 \%$; in the third in $87.5 \%$, in the fourth in $80.0 \%$ and finally in $50 \%$ of the fifth group (which was composed however only by 2 patients). This is an interesting data which emphasizes that FFA is not a pathology limited to the scalp and that can make us speculate that an advanced grade of FFA at the frontotemporal hairline (grade III, IV and V) could be associated with a more severe presentation in other district. Moreover, in about $30 \%$ of our cases the loss of the eyebrows was the first sign of the disease, preceding hairline recession. ${ }^{10}$

Hair loss in body also appeared to be more severe in patients with a higher degree of FFASI, with a percentage that was doubled from grade I (36.9\%) to grade V (100\%).

In accordance to the Literature, eyelash alopecia was present only in a few subjects. Noninflammatory facial papules had a low prevalence and were present only in 3 subjects, with no significant difference between women and men; this data is in contrast with OrmaecheaPérez case series, ${ }^{11}$ in which it is reported that FFA male patients have a higher prevalence of non-inflammatory papules than female patients.

\section{FFA: trichoscopy and histopathology}

Trichoscopic features of our patients were consistent with those reported in the Literature; the absence of follicular ostia and the empty follicle are typical finding in trichoscopy of FFA, which is in fact classified as a scarring alopecia. Perifollicular erythema and hyperkeratosis are also common characteristics of this disease that share this trichoscopic features with LPP, even though in the latter these two findings are usually reported to be more accentuated than in FFA. In our patients, perifollicular erythema and hyperkeratosis were present in $63.0 \%$ and $55.5 \%$ respectively, and in most cases, they were associated with subjective symptoms among which the most frequent was pruritus $(40.3 \%){ }^{12}$

The typical clinical and trichoscopic features of FFA allow clinicians to make diagnosis in most cases without the need of invasive diagnostic techniques; according to this, also in our sample the majority of patients have a clinical diagnosis $(n=83)$. However, in some cases, such as in the 8 men and in some women, mostly premenopausal, there were the need of a histological confirmation because the diagnosis was uncertain. ${ }^{8}$ 
Most patients who underwent biopsy had an overlapping with AGA or FPHL, which can mask FFA and delayed its diagnosis; in the other cases the histologic confirmation was necessary because patients had an initial form of the disease with a small recession of the frontotemporal hairline, which could be interpret as ophiasis. ${ }^{10}$ In all the specimens of FFA, mild lymphohistiocytic inflammatory infiltrate and lamellar fibrosis mainly located at the isthmus and infundibulum area of the hair follicle were observed. Histopathological features of AGA were present in 4 out 8 male patients, whereas FPHL in 13 out of 111 female patients.

\section{FFA and thyropathy}

In our sample, thyroid disease was reported by $18.6 \%$ of patients with FFA and all of them were female; this number is very high if compared with the mean prevalence of thyroid dysfunction in Europe (4\%). ${ }^{13}$

In the last few years it has been supposed a role of thyroid hormones in maintaining hair follicle immune privilege, which is lost in FFA, a disease where the hair follicle is attacked by an immune-mediated inflammatory infiltrate, characterized by a prevalence of CD8+ T lymphocytes. ${ }^{14}$

However, in the Literature, the association between FFA and thyroid dysfunctions in male patients is not so frequent, and also in our sample no man was affected by hypo or hyperthyroidism. ${ }^{13}$

\section{FFA and $L S$}

An interesting aspect found in our sample was the association between FFA and genital LS. In fact, 15 out of 119 FFA patients $(10.61 \%)$ were affected by concomitant genital LS (in particular, 2 male patients and 13 female patients). Among these 15 subjects, 5 patients presented both genital LS and cutaneous LP in addition to FFA.

LS prevalence is about $3 \%$ in general population, and it is considered a rare disease. ${ }^{15}$

Currently, the association between these two conditions had been rarely reported in the Literature.

To the best of our knowledge, there are 4 cases of concomitant FFA and LS reported yet. In 1996, Feldmann et al. described two postmenopausal FFA patients, among which one of them presented simultaneous vulvar LS. ${ }^{16}$ Later, in 2012, MacDonald et al. studied 60 FFA patients: concomitant vulval LS was described in one patient. ${ }^{17}$ Then, in a 2014 multicenter review of 355 patients with FFA, LS was found in one patient. ${ }^{5}$ Recently, Starace et al. 
described 65 Caucasian female patients with FFA, among which only one patient was also affected by genital LS. ${ }^{10}$

The higher prevalence of genital LS in our FFA patients lead to some speculations about a possible common pathogenic mechanism underlying both diseases.

FFA and LS are two chronic inflammatory conditions, whom pathogenesis is still not completely understood. First of all, both diseases show familial occurrence. Sherman et al. described that $12 \%$ of LS patients have a first-degree relative with the same condition, ${ }^{18}$ while a positive family history has been related in approximately $8 \%$ of FFA cases in the Literature. $^{3}$

Both FFA and LS present predisposal genetic factors as important pathogenic components. However, no genes appear to be shared between the two conditions. HLA-DQ7 seems to play a main role in LS susceptibility, ${ }^{3}$ as well as HLA-DR12 and haplotype DRB $1 * 12 / D Q B 1 * 0301 / 04 / 09 / 010 .{ }^{19}$ On the other hand, FFA genetic predisposition has not been systematically determined. HLA-B*07:02 has been recently described as a predisposing genetic factor of FFA. ${ }^{3}$

Environmental factors possibly related to LS include chronic irritant exposure to urine, traumatic stimuli, infections, such as Epstein-Barr virus and Borrelia, hormones and oxidative stress. $^{20}$

Also in FFA, irritant stimuli have been postulated as a possible trigger factor. In particular, sunscreen products and skin care product usage in patients with positive patch test to their ingredients seems to be associated with FFA. ${ }^{21}$

Furthermore, both conditions are frequently associated with other autoimmune diseases. The most common autoimmune conditions associated with LS are thyroiditis, AA, vitiligo and pernicious anemia. ${ }^{20}$ Also FFA patients are frequently affected by autoimmune disorders, mainly autoimmune thyroiditis. ${ }^{17}$

Considering the higher frequency of autoimmune comorbidities in both LS and FFA, it is possible to hypothesize an autoimmune pathogenesis in both conditions. However, the molecular target of the autoimmune process is still unknown.

Recently, serum extracellular matrix protein 1 (ECM1) antibodies have been detected in LS patients, both male and female. ${ }^{23,24}$ ECM1 a glycoprotein that acts as "biological glue", contributing to the organization of collagen fibers in the dermis. ${ }^{23}$ Indeed, ECM1 binds proteoglycans, in particular prelacan, that bind to fibronectin, laminin, type IV collagen, fibulin 2, dystroglycan, platelet-derived growth factor, fibroblast growth factor 7 and fibroblast-growth- factor-binding protein. Thus, disruption of these normal associations by 
antibodies to ECM1 could account for the histopathological abnormalities present in LS. ${ }^{23}$ ECM1 has been implicated in other fibrotic disorders, ${ }^{25}$ at the best of our knowledge it was never investigated in FFA, however as it is expressed in outer root sheath of hair follicles, we maight speculate that this protein may represent a target antigen for autoimmune process also in FFA.

Further studies are needed for a better understanding of the nature of the association between LS and FFA; because of the high prevalence of genital LS found in our sample, we suggest to investigate LS in all FFA patients, as it is often scantly symptomatic in early phase. 


\section{References}

1. Kossard S. Postmenopausal frontal fibrosing alopecia. Scarring alopecia in a pattern distribution. Archives of Dermatology. 1994;130(6):770-774.

2. Tolkachjov S, Chaudhry H, Camilleri M, Torgerson R. Frontal fibrosing alopecia among men: A clinicopathologic study of 7 cases. $J$ Am Acad Dermatol. 2017;77(4):683-690.e2.

3. Tziotzios C, Petridis C, Dand N, et al. Genome-wide association study in frontal fibrosing alopecia identifies four susceptibility loci including HLA-B*07:02. Nat Commun. 2019;10(1):1150.

4. Ho A, Shapiro J. Medical therapy for frontal fibrosing alopecia: A review and clinical approach. J Am Acad Dermatol. 2019;81(2):568-580.

5. Vano-Galvan S, Molina-Ruiz A, Serrano-Falcon C, et al. Frontal fibrosing alopecia: A multicenter review of 355 patients. J Am Acad Dermatol. 2014;70(5):AB90.

6. Holmes S, Ryan T, Young D, Harries M. Frontal Fibrosing Alopecia Severity Index (FFASI): a validated scoring system for assessing frontal fibrosing alopecia. $\mathrm{Br} J$ Dermatol. 2016;175(1):203-207.

7. Zhang M, Zhang L, Rosman IS, Mann CS. Frontal fibrosing alopecia demographics: a survey of 29 patients. Cutis. 2019; 103 (2): E 16-E22

8. Kanti V, Constantinou A, Reygagne P, et al. Frontal fibrosing alopecia: demographic and clinical characteristics of 490 cases. J Eur Acad Dermatol Venereol. 2019;33(10):1976-1983.

9. Navarro-Belmonte M, Navarro-López V, Ramírez-Boscà A, et al. Case series of familial frontal fibrosing alopecia and a review of the literature. J Cosm Dermatol. 2015;14(1):64-69.

10. Starace M, Brandi N, Alessandrini A, et al. Frontal fibrosing alopecia: a case series of 65 patients seen in a single Italian centre. J Eur Acad Dermatol Venereol.2019;33(2): 433-438.

11. Ormaechea-Pérez N, López-Pestaña A, Zubizarreta-Salvador J, et al. Frontal Fibrosing Alopecia in Men: Presentations in 12 Cases and a Review of the Literature. Actas Dermosifiliogr. 2016;107(10):836-844.

12. Fernandez-Crehuet P, Rodrigues-Barata AR, Vano-Galvan S, et al. Trichoscopic features of frontal fibrosing alopecia: results in 249 patients. J Am Acad Dermatol 2015; 72: 357-359.

This article is protected by copyright. All rights reserved 
13. Garmendia Madariaga A, Santos Palacios S, Guillen-Grima F, Galofre JC. The incidence and prevalence of thyroid dysfunction in Europe: a metaanalysis. J Clin Endocrinol Metab 2014; 99: 923-31.

14. Iorizzo M. and Tosti A. Frontal Fibrosing Alopecia: An Update on Pathogenesis, Diagnosis, and Treatment. Am J Clin Dermatol, 2019; 20(3), pp.379-390.

15. Virgili A, Borghi A, Toni G, et al. Prospective clinical and epidemiologic study of vulvar lichen sclerosus: analysis of prevalence and severity of clinical features, together with historical and demographic associations. Dermatology. 2014;228(2):14551.

16. Feldmann R, Harms M, Saurat JH. [Postmenopausal frontal fibrosing alopecia]. Hautarzt. 1996;47(7):533-6.

17. MacDonald A, Clark C, Holmes S. Frontal fibrosing alopecia: a review of 60 cases. $J$ Am Acad Dermatol. 2012;67(5):955-61.

18. Sherman V, McPherson T, Baldo M, et al. The high rate of familial lichen sclerosus suggest a genetic contribution: an observational cohort study. J Eur Acad Dermatol Venereol. 2010;24(9):1031-4.

19. Tran DA, Tan X, Macri CJ, et al. Lichen sclerosus: an autoimmunopathogenic and genomic enigma with emerging genetic and immune targets. Int J Biol Sci 2019;15(7):1429-1439.

20. Fistarol SK, Itin PH. Diagnosis and treatment of lichen sclerosus: an update. Am J Clin Dermatol. 2013;14(1):27-47.

21. Aldoori N, Dobson K, Holden CR, et al. Frontal fibrosing alopecia: possible association with leave-on facial skin care products and sunscreens; a questionnaire study. Br J Dermatol. 2016;175(4):762-7.

22. Tran DA, Tan X, Macri CJ, et al. Lichen Sclerosus: An autoimmunopathogenic and genomic enigma with emerging genetic and immune targets. Int $J$ Biol Sci. 2019;15(7):1429-1439.

23. Oyama N, Chan I, Neill SM, et al. Autoantibodies to extracellular matrix protein 1 in lichen sclerosus. Lancet. 2003;362(9378):118-23.

24. Edmonds EV, Oyama N, Chan I, et al. Extracellular matrix protein 1 autoantibodies in male genital lichen sclerosus. Br J Dermatol. 2011;165(1):218-9.

25. Tziotzios C, Stefanato CM, Fenton DA, et al. Frontal fibrosing alopecia: reflections and hypotheses on aetiology and pathogenesis. Exp Dermatol. 2016;25(11):847-852. 


\section{Legend to figures}

Figure 1. Eyebrow involvement in Frontal fibrosign alopecia: A) A 56 years-old female patient presenting with alopecia of the lateral aspects of eyebrow; B) Trichoscopical examination reveals area of absence of hair follicles, white dots and few yellow dots; perifullicular erythema and hyperkeratosis were not present. C) A 64 years-old female patient presenting with marked and diffuse eyebrow rarefaction; D) Trichoscopy shows absence of perifollicolar erythema and of perifollicular hyperkeratosis, several white and yellow dots are present; E) A 71 years-old female patient with complete eyebrow alopecia; F) At the trichoscopy presence of only one hair follicle; white and yellow dots are also present.

Figure 2. A 76 years-old patients affected by Frontal fibrosing alopecia A) fronto-teporal hairline recession with some lonely hair; B) At hystopathological examination, presence of lymphohistiocytic infiltrate located in the upper part of the hair follicle in the superficial dermis (Hematoxilin \& eosin, 40X); C) Ivory-white plaques with glistening surface affecting the vulva, with partial fusion of labia majora and labia minora; D) Elastic fibers (purplish black) appear dysorganized in the perifullicolar dermis (Weigert, 20X). 

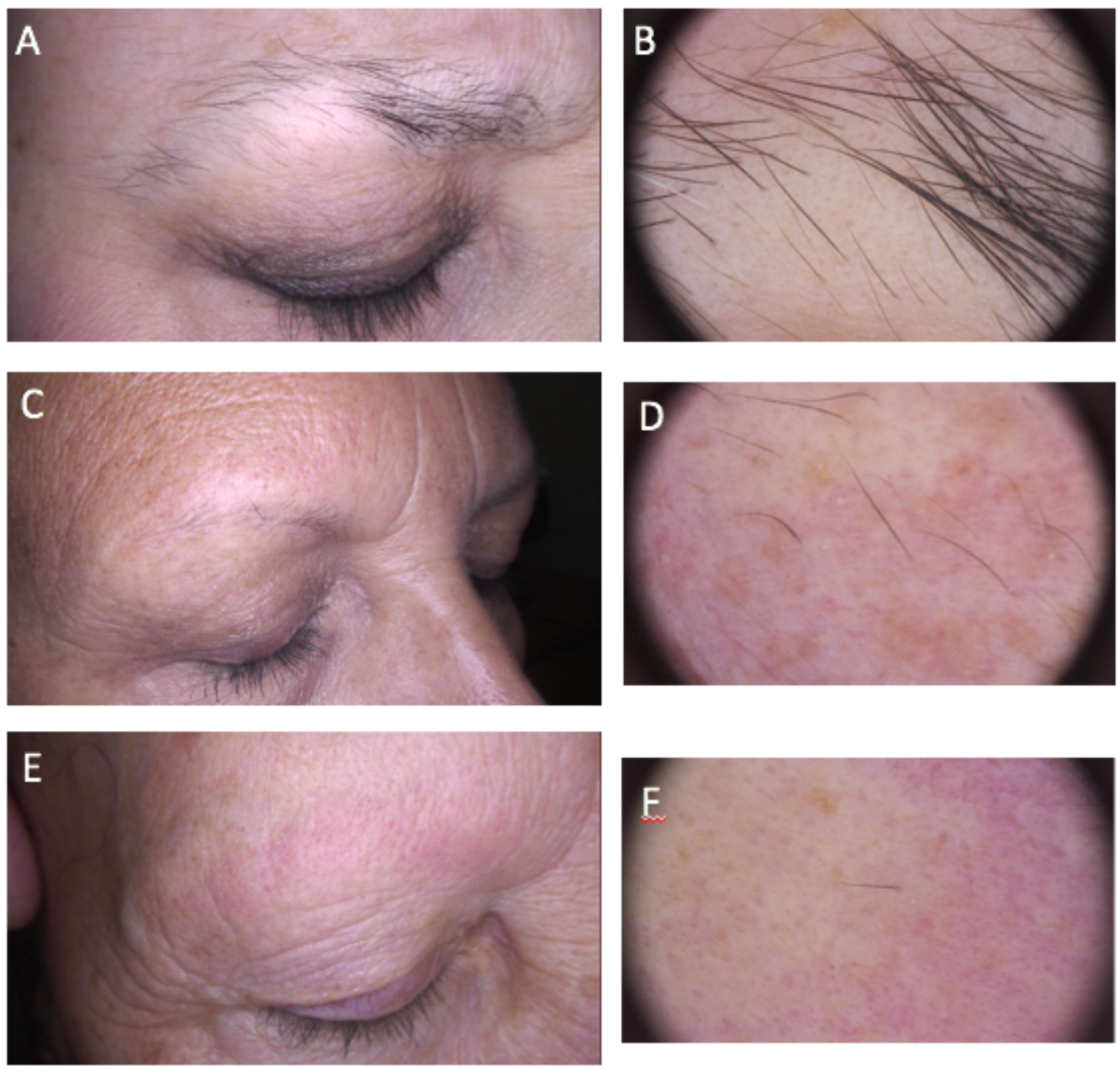

jocd_13573_f1.tiff 

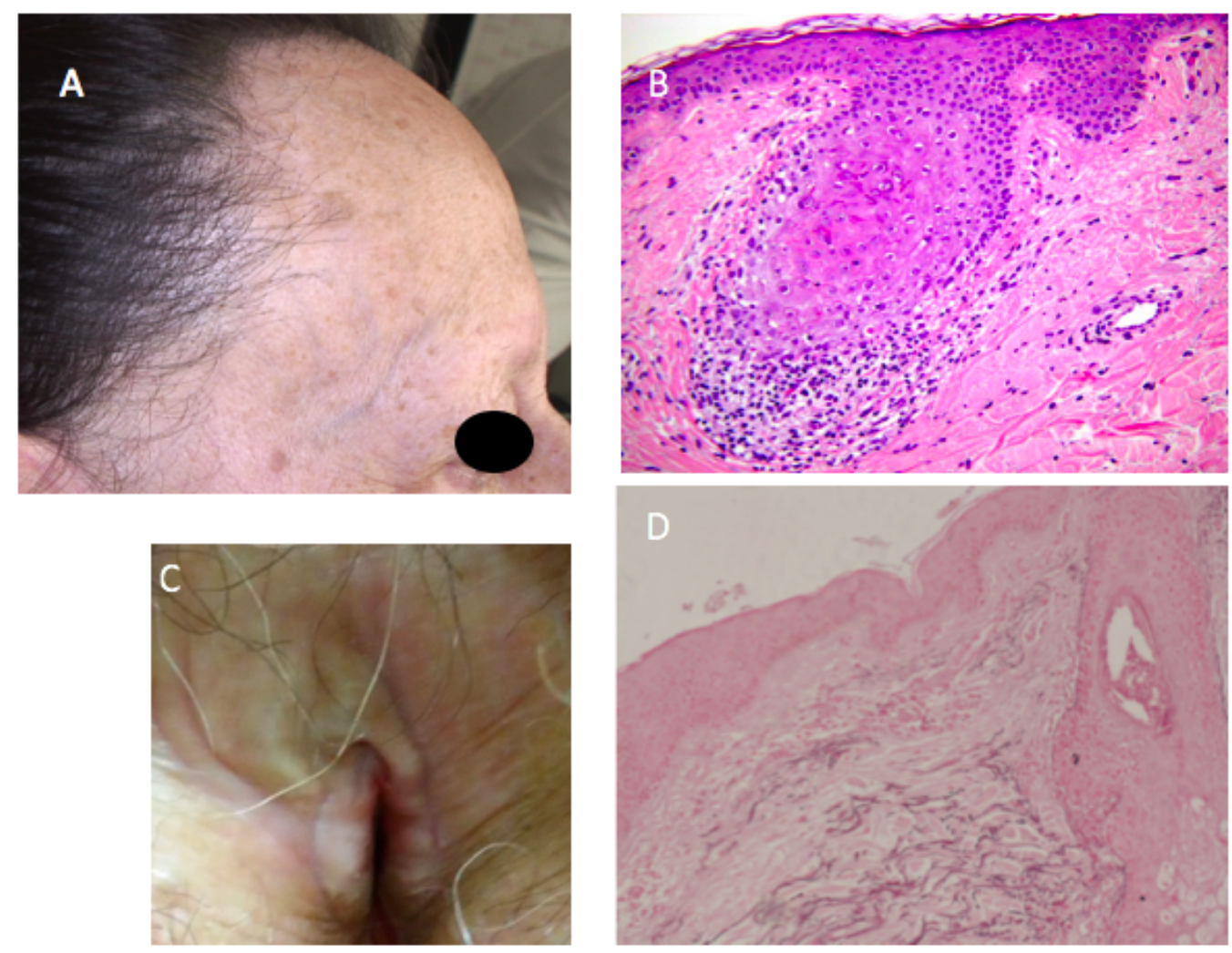

jocd_13573_f2.tiff 